\title{
Identification of Auto-antibody Producing Cells by Observing Antigen-antibody Reaction on the Cell Surface
}

\author{
Atsughi Horiuchi, Yuji Okamoto, Koichi Morita, \\ Shicemasa Sawada and Ichita Amaki \\ The First Department of Internal Medicine, Nihon Universiy \\ School of Medicine, Tokyo
}

\begin{abstract}
Horlechi, A., Okanoto, Y., Morita, K., SAwada, S. and Akaki, I. Identification of Auto-antibody Producing Cells by Observing Antigen-antibody Reaction on the Cell Surface. Tohoku J. exp. Med., 1972, 107 (1), 73-86. - Identification of the auto-antihody producing cells was attempted by the immunocyte adherence between the antigen coated erythrocytes and the autoantibody producing cells in the peripheral blood, lymph node and spleen of the cases with autoimmune diseases. The reaction was observed under a phase contrast mirroscope. The methods available for differentiation of antibody producing cells from antibody fixed colls in the on-cell-surface reaction were tested and discussed. The appearance rate of the auto-antibody producing cell was 1 to 3 per cent in the periphera! 1ymphocytes and 2 to 10 per cent in the lymph node cells. There was no correlation between the serum antibody titer and the number of positive cells. It has been revealed that the positive reaction of SLE is specifically inhibited by anti-Ig(t ( $z^{-c h}$-cin specific) serum and that of RA is inhibited by anti-IgM (/ antibody producing cells of SLE and RA produce $\operatorname{Ig}(;$ and $\operatorname{IgM}$ antibodies, respectively. The present method is based on the "virgin antibody" named by the present authors in 1969 , that is, the antibody just produced from the antibody producing cells and not yet combined with antigen. ___ auto-antibody producing cell; autoimmune disease; immunocyte adherence; virgin antibody
\end{abstract}

The mechanism of development of autoimmune disease remains still unknown in spite of earnest debates in recent years. It is doubtless, however, that the autoantibody from the auto-antibody producing cells is the direct cause of such diseases. The autoimmune diseases are usually incurable, thus indicating that, once provoked, abnormal production of antibody due to proliferation of the antibody producing cells cannot be controlled. This fact led Dameshek (1967) to discuss their similarity to leukemia.

To cure autoimmune disease, it might be necessary to depress a proliferation of such cells or their function, but no decisive method for either of them has yet been established. In order to investigate autoimmune diseases and develop the therapies for them, studies are needed on the morphology, varieties, functions, distribution, kinetics, and above all, identification of the auto-antibody producing 
cell.

As they are functioning cells, the method to identify them should be based on their function. A reliable identification method of the antibody producing cell by gross observation is the agar plaque method (Jerne and Nordin 1963), which is based on the hemolysis of erythrocytes around the antibody producing cell. But the present authors have contrived a qualitative and quantitative method by means of phase contrast microscopy (Irimajiri et al. 1969), which is based on the immunocyte adherence, i.e., agglutination of the antigen coated erythrocytes around the auto-antibody producing cell. The attempt was made to apply this method to identify the auto-antibody producing cells.

In the previous experiment (Amaki et al. 1969), latex particles coated with the antigen were brought to reaction in a tube with the cells of peripheral blood or bone marrow. As a result, immunocyte adherence between the antigen-latex particles and the antibody producing cells was observed. This method required relatively simple procedures, but possible concomitance of non-specific reactions could not be excluded.

The present paper is concerned with the methodology and results of a series of experiments to detect the auto-antibody producing cell, using erythrocytes instead of latex as the antigen coated particles.

\section{Materials and Methods}

Test subjects

Ninety-two cases were examined in total; 4 cases of autoimmune hemolytic anemia (AIHA), 21 cases of systemic lupus erythematosus (SLE), 46 cases of rheumatoid arthritis ( $R A$ ), 5 cases of Hashimoto's disease, and 16 cases of normal adults.

Antigen

a) For AIHA, the patient's erythrocytes were used after the treatment with bromelin or ficin.

b) For SLE, both DNA and histone (Signia Co.) were used as antigens. DNA was restricted to the one with a peak at $260 \mathrm{~m} \mu$ and the protein content of $0.7 \%$ and $50 \gamma$ of such DNA were used to coat $1 \mathrm{ml}$ of the $1 \%$ tanned sheep erythrocytes. Two kinds of erythrocytes of different shape were used in order to know if there was such a cell as the so-called double producer of antibodies by forcing the auto-antibody producing cells to react with the two different antigens simultaneously.

c) For RA, $50 \gamma$ of human gamma globulin (Fr. II, Nutrit. Biochem. Co.) were used after heat denaturation at $63^{\circ} \mathrm{C}$ for 15 minutes to coat $1 \mathrm{ml}$ of the $1 \%$ tanned sheep erythrocytes.

d) For Hashimoto's disease, the commercially available thyroglobulin coated sheep erythrocytes (prepared by the Fuji Zoki Pharm. Ltd.) were used as the antigen coated particles.

\section{Preparation of the antibody producing cell}

Twenty to thirty milliliters of peripheral blood collected from the patient's vein were immediately defibrinated and then centrifuged at $100 \times \mathrm{g}$ for 10 minutes. The supernatant, rich in lymphocytes, thus obtained was recentrifuged at $200 \times \mathrm{g}$ for 10 minutes. The sediment was washed twice with TC-199 solution by centrifusing at $200 \times \mathrm{g}$ for 10 minutes.

The bone m arrow cells or the lymph node and spleen cells teased out in the TC-199 solution were also washed in the same way. Each of the cell suspensions was subjected to 
the final adjustment through the TC-199 solution so that the cell count might remain within the range of 10,000 to $20,000 / \mathrm{cmm}$.

\section{Antigen-an tibcdy reaction}

To $0.5 \mathrm{ml}$ of the cell suspension which had been incubated at $37^{\circ} \mathrm{C}$ for 30 minutes was added $0.5 \mathrm{ml}$ of the antigen coated erythrocyte suspension. The mixture was kept at room temperature for about 30 minutes, with gentle shake, and then one drop of the mixture was put on the slide glass, enclosed with a cover glass, and observed with a phase contrast microscope.

A cell to which adhered four or more antigen coated erythrocytes was regarded as positive cell (the antibody producing cell), because such a cell was hardly seen in normal controls. The percentage of the positive cells was calculated in 200 to 500 lymphocytes.

Control

The following procedures were carried out to provide the basis for comparison with the foregoing experimental results.

a) The reaction of the peripheral lymphocytes of the normal adults to the antigen coated erythrocytes was observed by the same method.

b) Sheep erythrocytes without antigen were made to react upon the patient's lymphocytes to see if there existed cells producing any heterophil antibody to sheep erythrocytes.

c) In order to confirm whether these were really caused by an antigen-antibody reaction, the surface of the antibody producing cells was preliminarily blocked with the same antigen as was used to coat the erythrocytes and then their reaction to the antigen coated erythrocytes was observed.

d) With a view to studying the types of immunoglobulin produced by the cells, the surface of the cells was blocked with anti-IgG serum ( $\gamma$-chain specific) or anti-IgM serum ( $\mu$-chain specific), and then the cells were made to react with the antigen coated erythrocytes.

e) In order to demonstrate that the immunocyte adherence was not the non-specific adsorption on cell surface, but the specific reaction between the antigen coated erythrocytes and the antibody produced by the cell, the cells, of which the surface antibody had been blocked with the antigen, were incubated at $37^{\circ} \mathrm{C}$ for 30 minutes to produce more antigen and then let them react with the antigen coated erythrocytes.

\section{Results}

\section{1. $A I H A$}

Figs. 1 (a) and (b) show the positive cell in the peripheral lymphocyte in a case of AIHA. The former represents the positive reaction between the patient's erythrocytes treated with bromelin and her own lymphocyte, and the latter represents the positive reaction when the erythrocytes were treated with ficin.

Fig. 2 demonstrates a comparison of the positive cell percentage between the spleen cells and peripheral lymphocytes in an 18 years old female with AIHA, who underwent splenectomy after the administration of prednisolone over a long period of time. In the figure, the ordinate indicates the percentage of the positive cells and the abscissa indicates the number of the antigen coated erythrocytes adhering to the cell. Providing that a cell which four or more erythrocytes adhered to was positive, there was no difference in percentages of positive cells between spleen cells and peripheral lymphocytes. This may be attributed to the fact that a large dose of prednisolone such as $500 \mathrm{mg}$ was administered during the operation. 


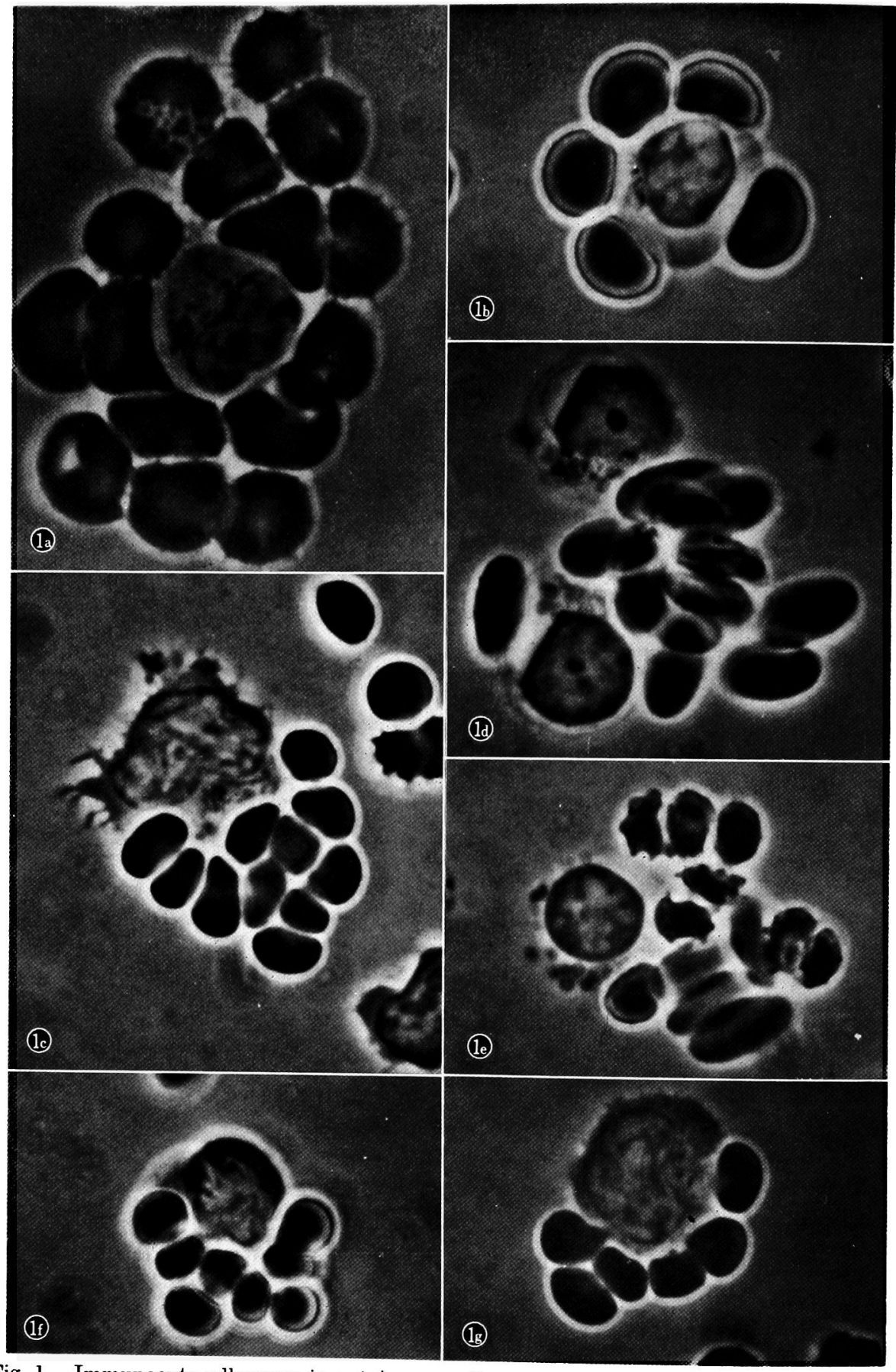

Fig. 1. Immunocyte adherence in autoimmune diseases. (a) and (b) are peripheral lymphocytes in AIHA. The patients erythrocytes are treated with bromelin (a) or ficin (b). The lymph node cells reacted with DNA coated SRBC (c) and histone coated llama erythrocytes (d) in SLE. The double producer cell in SLE (e). The peripheral lymphocytes reacted with denatured $\gamma$-globulin coated SRBC in RA (f) and (g). 


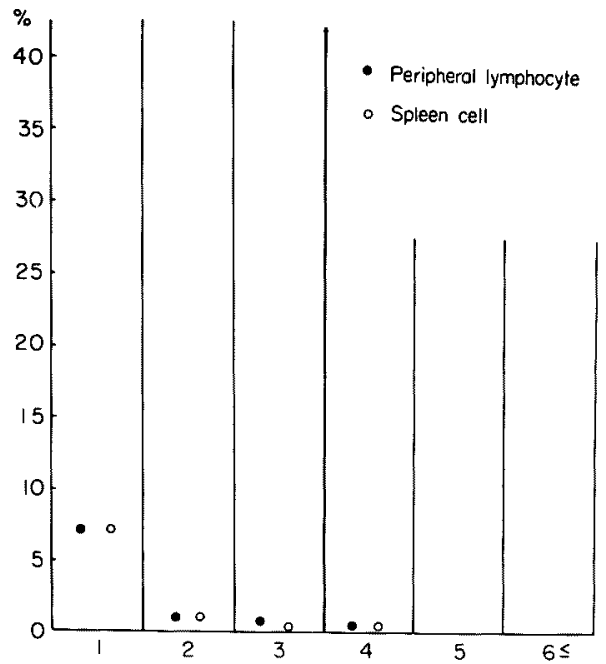

Fig. 2. Immunocyte adherence in AIHA case (18-years-old female).

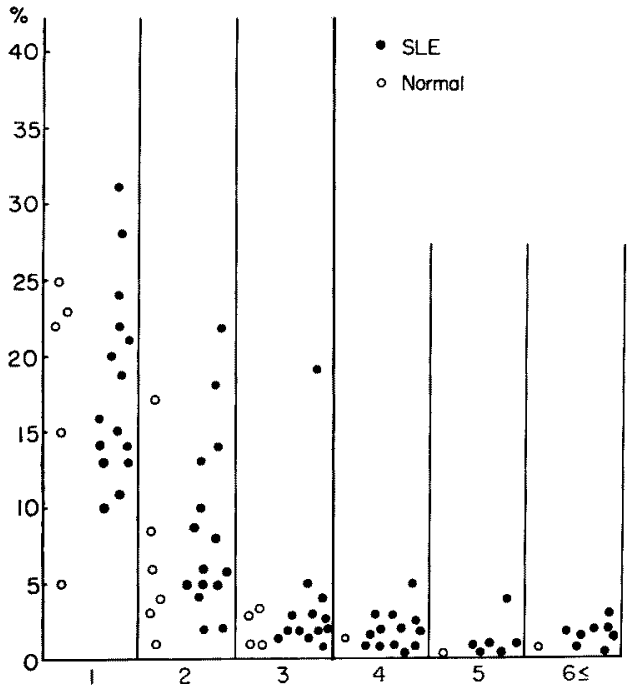

Fig. 3

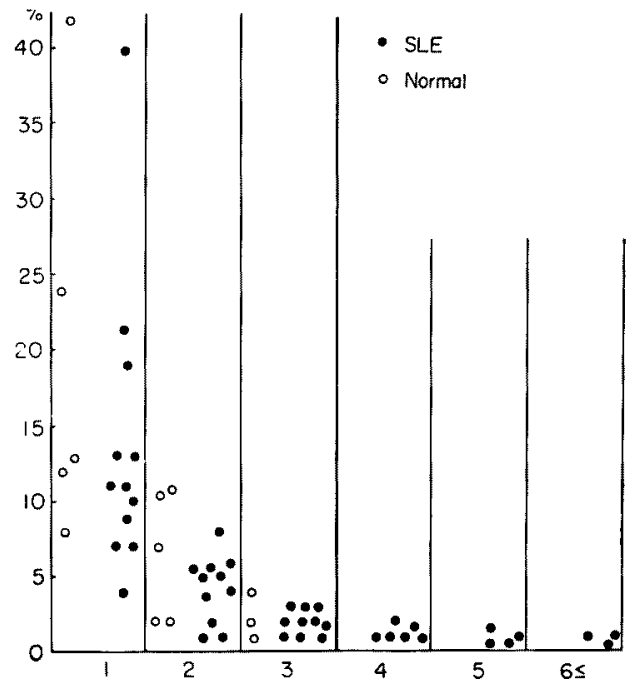

Fig. 4

Fig. 3. Immunocyte adherence to DNA in SLE and normal cases.

Fig. 4. Immunocyte adherence to histone in SLE and normal cases.

\section{SLE}

Of the peripheral lymphocytes from SLE cases, the cells positive to the DNA antigen are presented in Fig. 1 (c) and those to the histone antigen in (d). The elliptical erythrocytes are the llama's coated with histone. Fig. 1 (e) shows the cells regarded as the double producer cells, which were quite rarely encountered in 500 lymphocytes. 


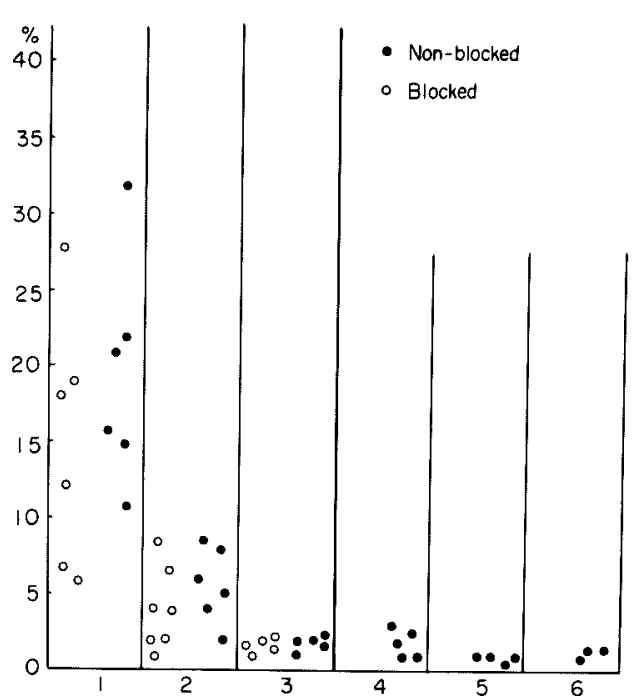

Fig. 5

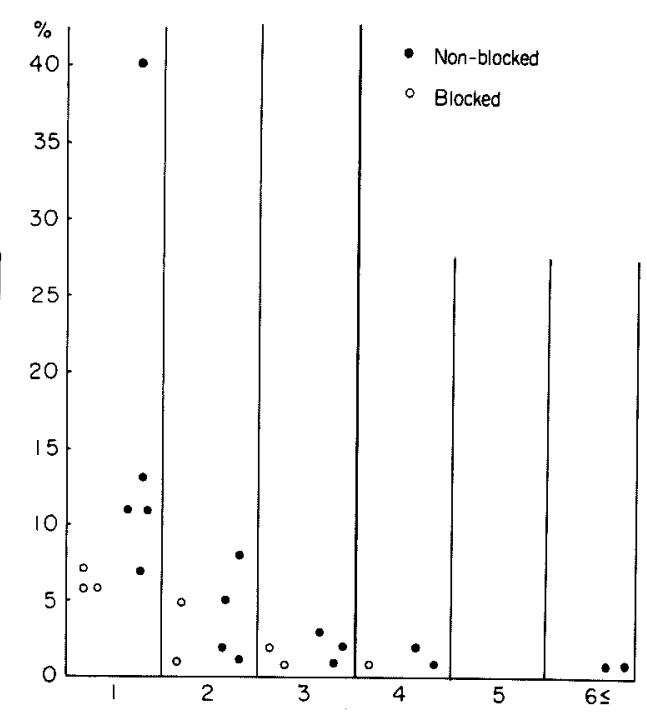

Fig. 6

Fig. 5. Immunocyte adherence to DNA after being blocked with DNA in SLE cases.

Fig. 6. Immunocyte adherence to histone after being blocked with histone in SLE cases.

Comparison was made on the reaction of lymphocytes to DNA and histone between SLE cases and normal adults, of which the results are demonstrated in Figs. 3 and 4. As to the reaction to DNA, the positive cells appeared far more frequently in the SLE cases, although there were normal cases which showed cells with more than four erythrocytes. As to the reaction to histone, there were no positive cells in the normal cases.

Fig. 5 gives the result of an experiment in which the surface of the antibody producing cells was preliminarily blocked with DNA and then made to react to the DNA coated erythrocytes. Fig. 6 shows a similar experiment with histone. A definite difference was disclosed between the blocked and non-blocked groups, as no positive cells were demonstrated in the blocked group. This fact evidences that the immunocyte adherence in this experiment is due to an antigen-antibody reaction.

Immunoglobulin on the surface of the antibody producing cells was blocked with either anti-IgG serum ( $\gamma$-chain specific) or anti-IgM serum ( $\mu$-chain specific) and then the cells were made to react to the antigen coated erythrocytes. Fig. 7 shows the cells which were blocked with anti-IgG serum and made to react to the histone coated llama erythrocytes, and Fig. 8 shows the one blocked with anti-IgM serum. As a result, it was found that the reaction was inhibited with anti-IgG serum but hardly with anti-IgM serum.

In cases of DNA coated sheep erythrocytes, the reaction of cells was undoubtedly inhibited with anti-IgG serum but not with anti-IgM serum.

Fig. 9 indicates the results in which the surface of the antibody producing cells was preliminarily blocked with the DNA or histone antigen, incubated at 


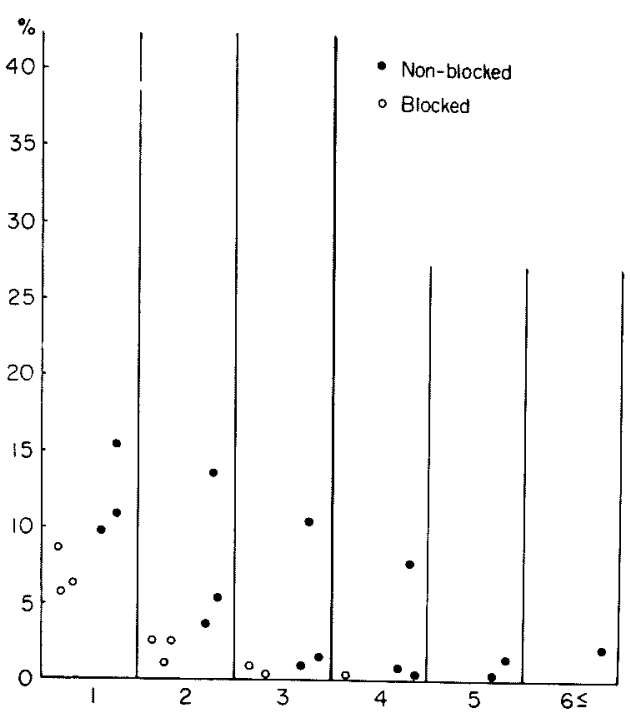

Fig. 7

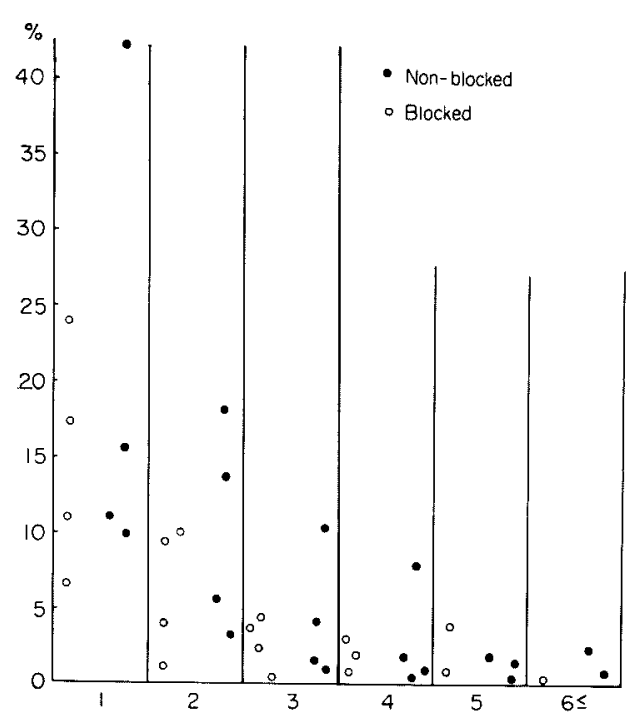

Fig. 8

Fig. 7. Immunocyte adherence after being blocked with anti-IgG serum in SLE cases.

Fig. 8. Immunocyte adherence after being blocked with anti-IgM serum in SLE cases.

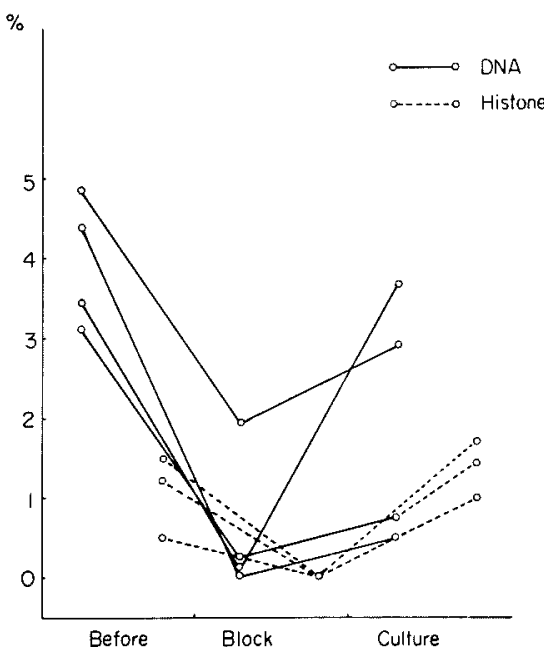

Fig. 9. Immunocyte adherence after culture of cells which were blocked with antigen in SLE cases.

$37^{\circ} \mathrm{C}$ for 30 minutes and let react upon the antigen coated erythrocytes. The positive cells were observed in the SLE cases, but not in the normal cases.

The reaction of the lymph node cells from a case of SLE was also observed; Fig. 10 gives the reaction to DNA and Fig. 11 that to histone. The reaction to either of the antigens was not blocked with anti-IgM serum but was blocked with anti-IgG serum. No substantial difference was demonstrated in the number of 


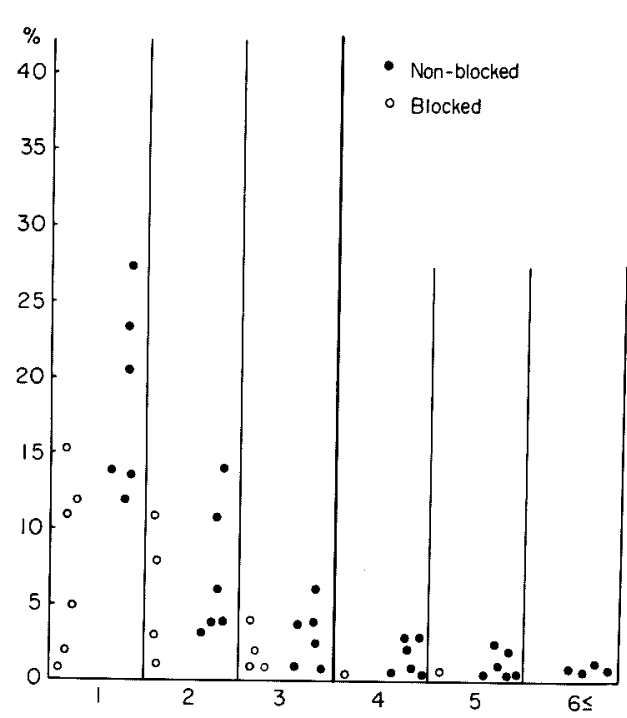

Fig. 10

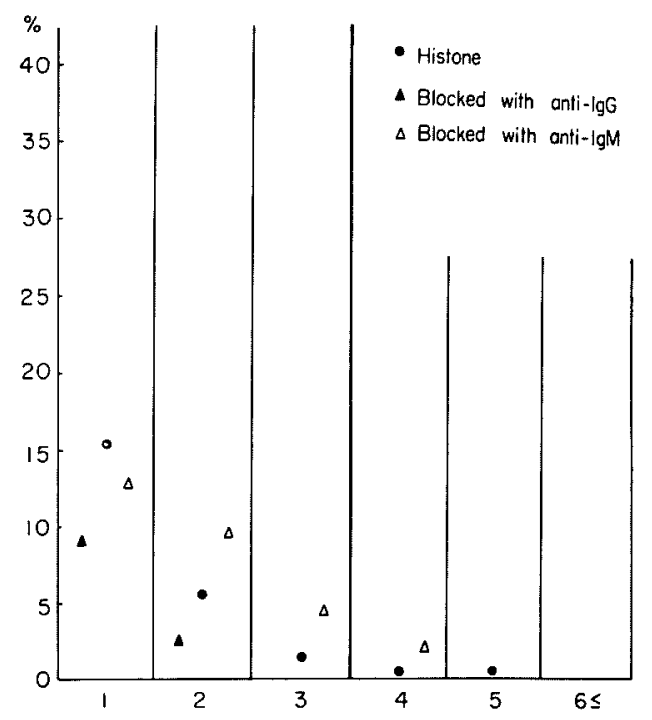

Fig. 11

Fig. 10. Immunocyte adherence to DNA in lymph node cells of SLE case (20-year-old, male).

Fig. 11. Immunocyte adherence to histone in lymph node cells of SLE case (20-year-old, male).

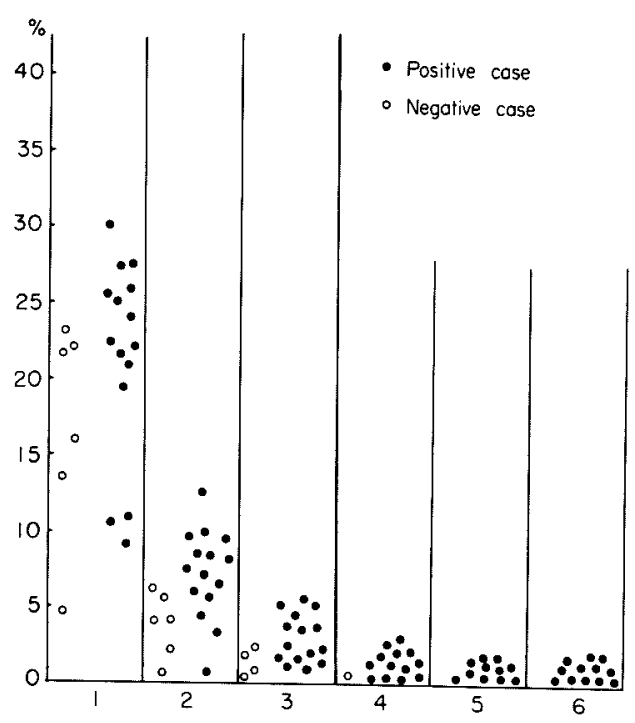

Fig. 12

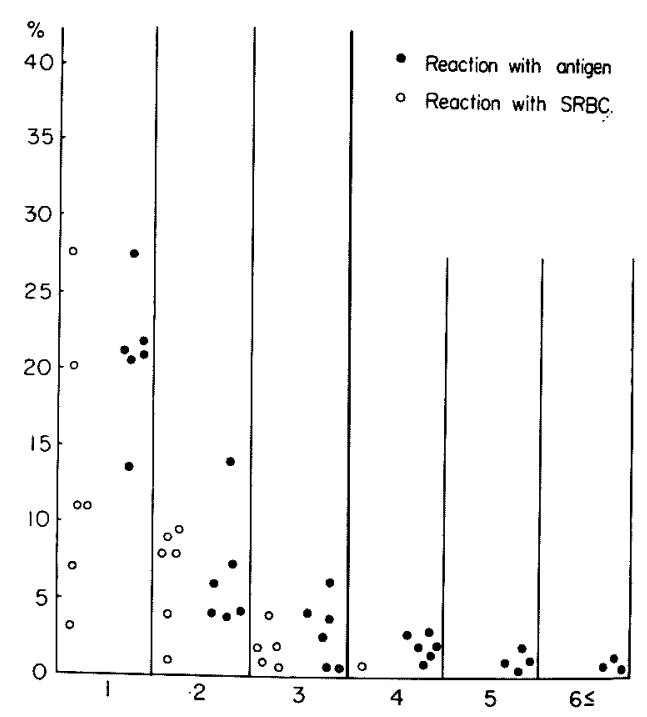

Fig. 13

Fig. 12. Immunocyte adherence in RA test positive and negative cases.

Fig. 13. Immunocyte adherence to $\mathrm{SRBC}$ in RA test positive cases. 


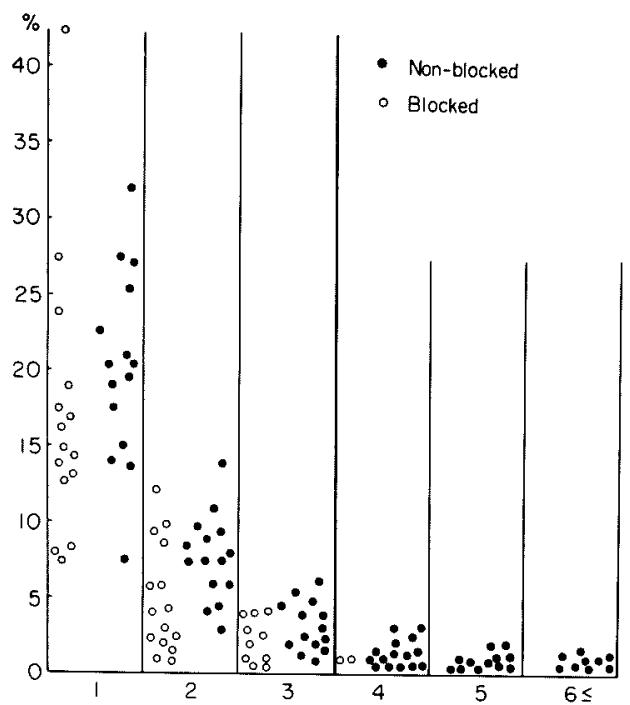

Fig. 14. Immunocyte adherence to $\gamma$-globulin in RA test positive cases after being blocked with $\gamma$-gloublin.

the positive cells between the lymph node cells and peripheral lymphocytes, the positivity rate being 2 to $10 \%$ for the former and 1 to $3 \%$ for the latter.

\section{3. $R A$}

Figs. 1 (f) and (g) illustrate the positive cells in the peripheral lymphocytes of RA. As in SLE, it was characteristic that the sheep erythrocytes coated with denatured $\gamma$-globulin adhered to one side of the cell.

Fig. 12 shows a comparison of the reaction to denatured $\gamma$-globulin between the RA cases and normal adults. Fifteen were taken at random out of 46 cases of RA and compared with 6 cases of normal adult. The positive cells were marked by significantly higher frequency in RA cases than in normals, although there was a normal case in which the positive cells were fairly demonstrated.

Fig. 13 shows the results of reactions of the peripheral lymphocytes of RA to the sheep erythrocytes with and without the antigen coating. The positive reaction was not due to heterophil antibodies.

Fig. 14 shows the results of the experiment in which the surface of the cells was blocked with the same antigen as used for the coating and then let react to the antigen coated erythrocytes. In the blocked group the positive cells almost disappeared. This finding clearly supports the view that this immunocyte adherence is due to an antigen-antibody reaction.

The results are shown of the reactions to the antigen coated erythrocytes after blocking with anti-IgG serum (Fig. 15) and with anti-IgM serum (Fig. 16). No remarkable inhibition took place with anti-IgG serum blocking, whereas many cases were inhibited with anti-IgM serum. 
A. Horiuchi et al.

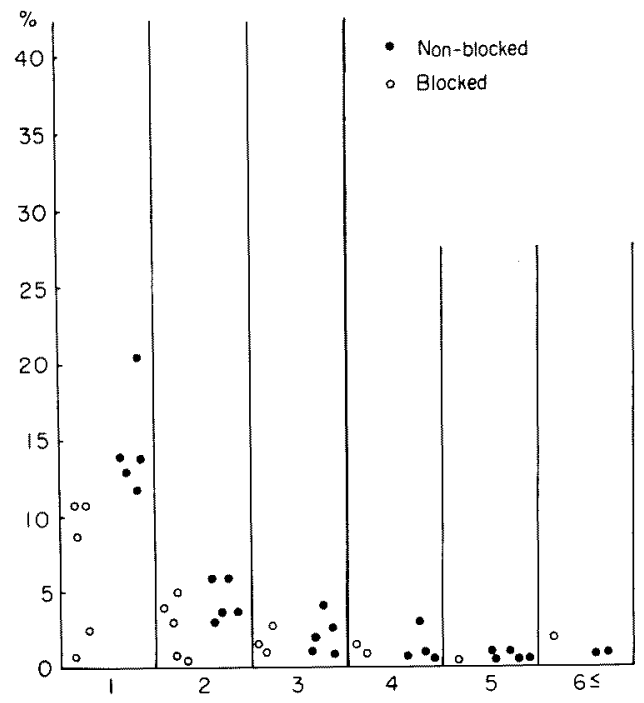

Fig. 15

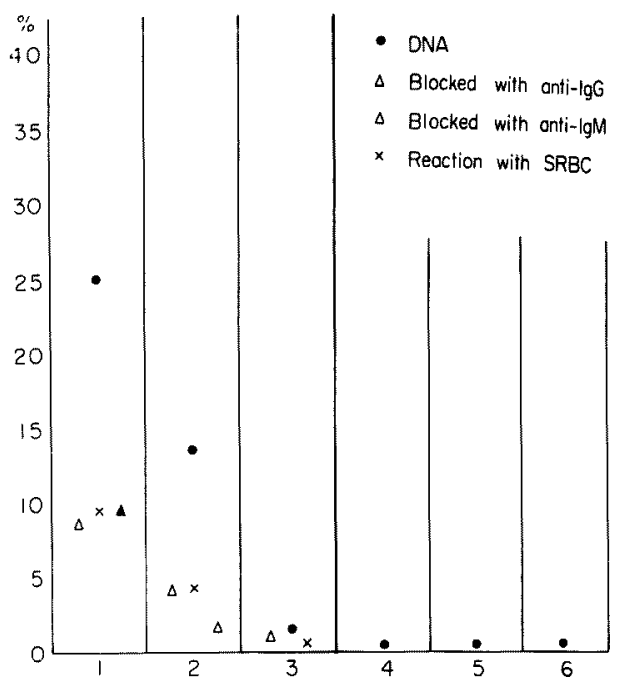

Fig. 16

Fig. 15. Immunocyte adherence after being blocked with anti-IgG serum in RA test positive cases.

Fig. 16. Immunocyte adherence after being blocked with anti-IgM serum in RA test positive ¿cases.

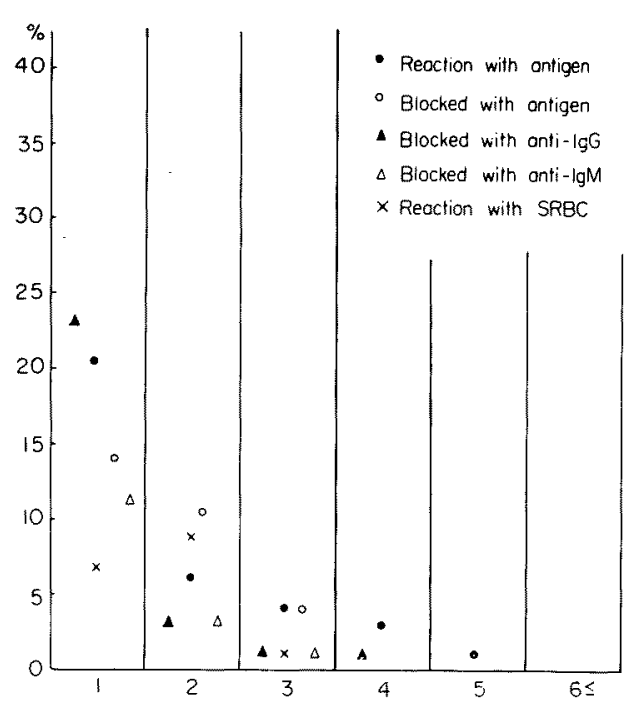

Fig. 17

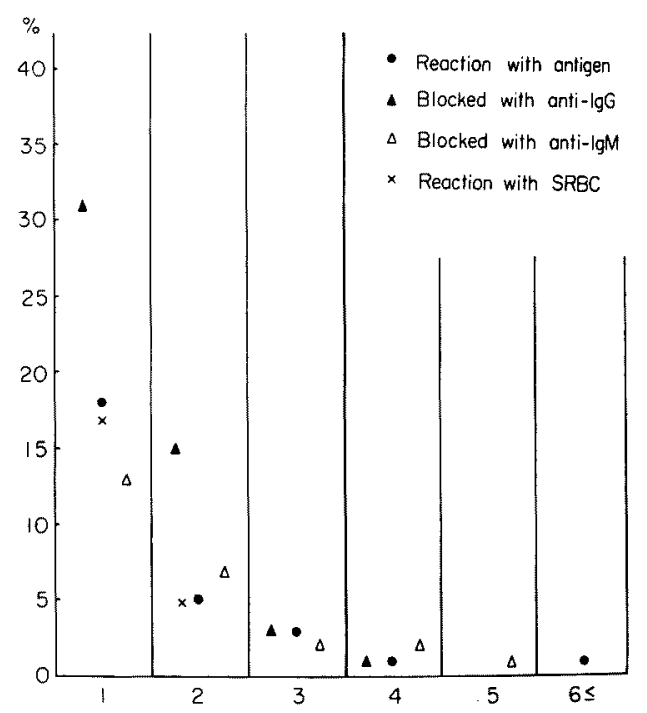

Fig. 18

Fig. 17. RA test positive case (20-year-old male, rheumatoid arthritis).

Fig. 18. RA test negative case (26-year-old male, normal adult).

Fig. 17 illustrates a case of $\mathrm{RA}$; the positive cells were not blocked anti-IgG serum but clearly blocked with anti-IgM serum, and also with the antigen. Fig. 18 shows a normal case in which the positive cells appeared. Some of the positive cells were inhibited with either anti-IgG or anti-IgM serum, but others were not. 


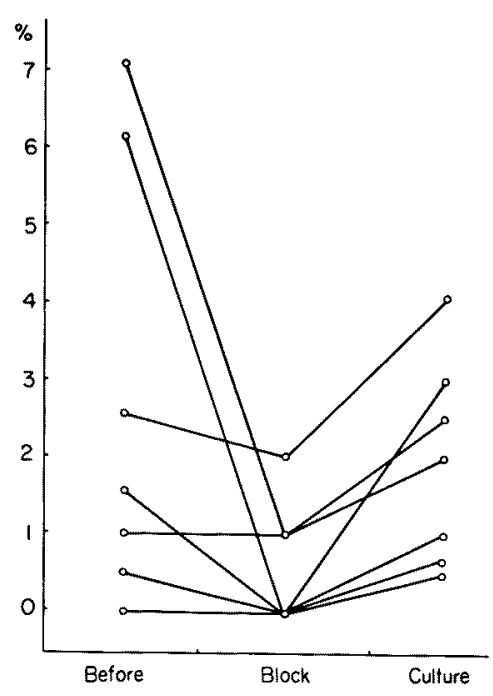

Fig. 19

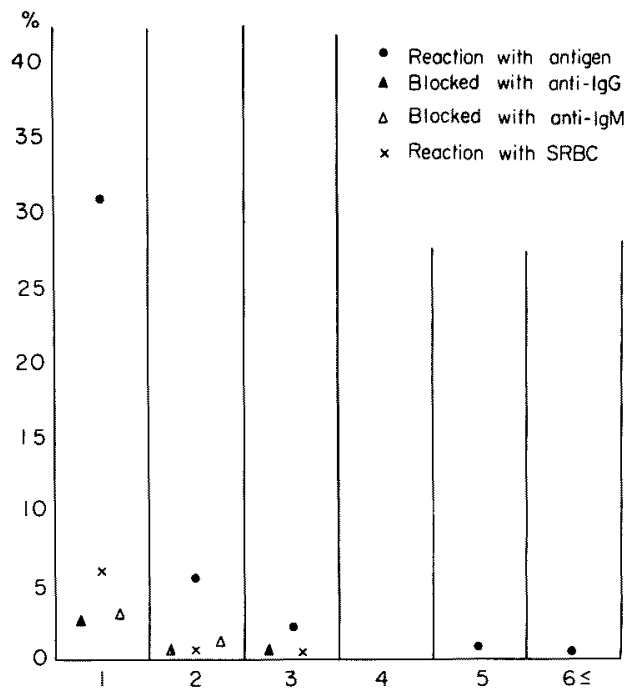

Fig. 20

Fig. 19. Immunocyte adherence after culture of cells which were blocked with antigen in RA cases.

Fig. 20. TA test positive case (56-year-old female, Hashimoto's disease).

Fig. 19 shows the results of an experiment in which the cells were blocked with the antigen and then, after incubation at $37^{\circ} \mathrm{C}$ for 30 minutes, made to the antigen coated erythrocytes. The positive cells came to be noted after the incubation, thus presenting a definite difference from the group that had undergone the blocking only.

\section{Hashimoto's disease}

As seen in Fig. 20, the positive cells among the peripheral lymphocytes of Hashimoto's disease were inhibited with anti-IgG and anti-IgM serum alike.

\section{Discussion}

Considerable efforts have been devoted in recent years to study on autoimmune diseases using the patient's serum or tissues as the material, but the cells in the peripheral blood or lymph node have been rarely used. In our previous work (Irimajiri et al. 1969) certain improvement was attempted on the agar plaque method by Jerne and Nordin (1963), which is a reliable procedure for the identification of antibody producing cells, and a new method was devised for qualitative and quantitiative observation of the antibody producing cells under a phase contrast microscope.

This method was based on the hemolysis of erythrocytes occurring around the antibody producing cell and the agglutination of erythrocytes to the cell, or immunocyte adherence. In this antigen-antibody reaction participates the anti- 


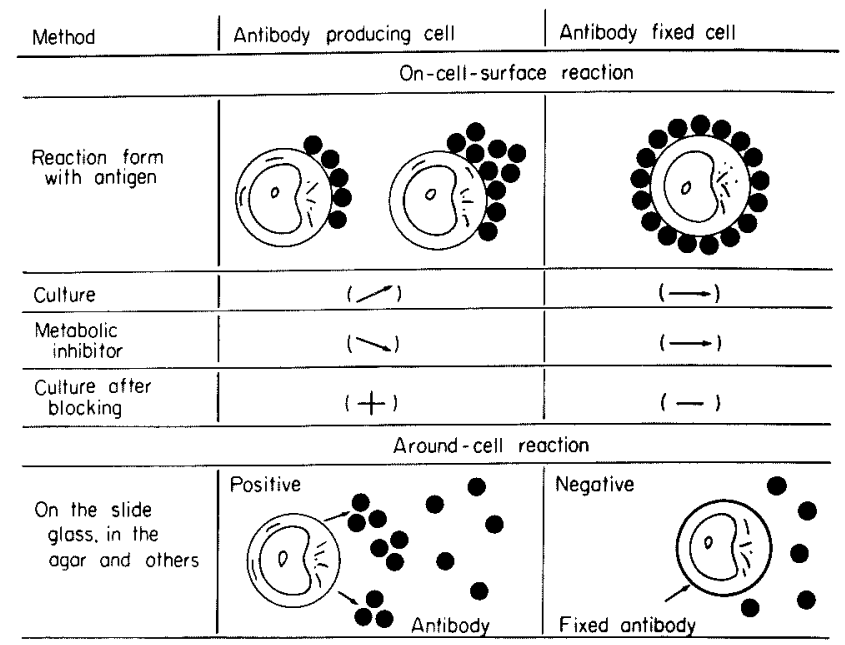

Fig. 21. Possibility of differentiation between antibody producing cell and antibody fixed cell.

body just yielded by the antibody producing cell, which we call "virgin antibody" (Amaki et al. 1969). The previous report (Amaki et al. 1969) was concerned with an identification method of the auto-antibody producing cell by using antigen coated latex particles. In this method there was a possibility that non-specific reactions might be included. Therefore, a larger particle, erythrocyte, was substituted for latex to exclud non-specific reactions as much as possible.

In the immunocyte adherence, most cases required differentiation of the antibody producing cell from the antibody fixed cell (cytophilic antibody). Fig. 21 illustrates the procedures for this differentiation. In the present study, these procedures were not all applied to the differentiation of the cells, but there is sufficient evidence to warrant that the positive cells were the antibody producing cells and not the antibody fixed ones.

It was found that, when the antigen coated erythrocytes adhered to an antibody producing cell, they formed characteristically a single layer or multiple layers on a part of the cell surface or that, when the cell in multiple layers. When the erythrocytes were lined circumferentially on the cell in a single layer, the possibility to be antibody fixed cell could not be ruled out. The site where the erythrocyte adhered was most often on the side near the Golgi area. This may be that the antibody production occurs mainly at the side a little lateral to the Golgi area and as the production is increased, the area for the antibody secretion is enlarged and gradually erythrocytes come to cover the whole surface of the cell doubly and triply.

Preliminary blocking of the cell surface with the antigen inhibited the appearrance of positive cells in the reaction between antibody producing cells and antigen coated erythrocytes. This finding affords an irrefragable proof that this is doubtlessly an antigen-antibody reaction. Further, when the blocked cells 
underwent incubation, positive cells reappeared. Thus, this fact confirms that those having produced the immunocyte adherence were the antibody producing cells.

The appearance rate of the positive cells in the immunocyte adherence was higher in the latex method than in the erythrocyte method. This may be interpreted as that the latex particles are more apt to cause a non-specific reaction. Although the appearance rate in the reaction to erythrocytes fairly varied from case to case according to the severity of the disease and the curative agents applied, it was 1 to $3 \%$ for the peripheral blood and 2 to $10 \%$ for the lymph node. There was almost no correlation between the appearance rate of positive cells and the antibody titer of serum on the same patient: e.g., there was a case in which the serum antibody was negative while the positive cells appeared in the reaction. This may support the idea of the virgin antibody.

In SLE the positive cells were inhibited chiefly with anti- $\gamma$ chain serum, and in RA they were inhibited with anti- $\mu$ chain serum. These facts imply that the immunoglobulin produced by the antibody producing cell is mainly IgG in SLE and in RA.

In some cases with SLE a few cells reacted to both antigens DNA and histone, while most cells reacted specifically to either of them. No particular relationship has been detected so far between reaction and the clinical findings, but further efforts will be directed to clarification on this respect.

When denatured $\gamma$-globulin or DNA was used as the antigen, the positive cells did appear in the normal controls, though at a very low rate, and this was seen especially when denatured $\gamma$-globulin was used. In such cases, the reaction was inhibited with anti-immunoglobulin in some cases but not in others. Among the inhibited cases, some were found to have an RA patient in the family. No appropriate interpretation could be given to the cases in which the reaction was not inhibited with anti-immunoglobulin.

Bach et al. (1970) have reported on the immunocyte adherence in the peripheral blood cells detected by a different method. They have also documented the appearance of positive cells in the normal control which were not inhibited with anti-immunoglobulin. It seems necessary to study these facts in the light of the rheumatoid factors.

The positive cells were also demonstrated in cases of AIHA and Hashimoto's disease, though the cases studied were not many. As for AIHA, the appearance rate of positive cells was compared between the spleen cells and peripheral lymphocytes, but no significant difference was demonstrated.

\section{The concept of the "virgin antibody"}

Various auto-antibodies have been demonstrated in autoimmune diseases, but they are hardly regarded as the direct cause of the diseases. In the case of AIHA, the auto-antibody adherence to erythrocytes in the first place, resulting in the positive Coombs' direct reaction, and if there is a surplus of the antibody, it 
appears in serum, resulting in the positive Coombs' indirect reaction.

From these facts, it is presumed that the produced auto-antibodies in autoimmune diseases immediately react to the antigens distributed widely and abundantly all over the body, and in the serum they appear in a very small quantity or in fairly modified forms, or exist as the so-called antigen-antibody complex.

In exploration of the auto-antibody in autoimmune diseases, it is important to study the antibody just produced and not yet combined with antigens. We have termed such an antibody as a "virgin antibody". The antigen-antibody reaction around the antibody producing cell is one of various methods for observation of the virgin antibody.

\section{Conclusion}

1. The identification of auto-antibody producing cells was attempted by making these cells in peripheral blood, lymph nodes and spleen of the cases with autoimmune diseases to react to the antigen coated erythrocytes.

2. According to this method, the cells and the antigen were brought to reaction in vitro and the immunocyte adherence was studied under a phase contrast micrscope. It was confirmed that the positive cells were not the antibody fixed ones, but the antibody producing cells.

3. The auto-antibody producing cells were observed in cases of AIHA, SLE, RA and Hashimoto's disease.

4. It is conceivable that this method could be applied, when further improved, to exploration of the entity of autoimmune disease, their diagnosis and treatment as a means of clinical immunology.

5. This method is based on the "virgin antibody" and its concept was discussed.

\section{References}

1) Amaki, I., Horiuchi, A., Irimajiri, K., Okamoto, Y., Morita, K. \& Hagihara, T. (1969) Identification of auto-antibody producing cell - observation of antigen-antibody reaction around the antibody producing cell. Clin. Immunol. (Jap.), 1, 431-440.

2) Bach, J.F., Delrieu, F. \& Delbarre, F. (1970) The rheumatoid rosette. A diagnostic test unifying seropositive and seronegative rheumatoid arthritis. Amer. J. Med., 49, 213-222.

3) Dameshek, W. (1967) Chronic lymphocytic leukemia - an accumulative disease of immunologically incompetent lymphocytes. Blood, 29, 566-584.

4) Irimajiri, K., Horiuchi, A., Okamoto, Y. \& Amaki, I. (1969) Studies on antibodyproducing cells by hemolytic plaque method and phase contrast microscopy. Blood, 33, 149-158.

5) Jerne, N.K. \& Nordin, A.A. (1963) Plaque formation in agar by single antibodyproducing cells. Nature, $140,405$. 